\title{
An Adaptive Energy-Efficient and Low-Latency MAC Protocol in Wireless Sensor Networks
}

\author{
Men Chaoguang ${ }^{1,2}$, Lu Yongqian $^{1}$, Zhang Huajian $^{1}$, and Li Guang ${ }^{1}$ \\ ${ }^{1}$ Research and Development Center of High Dependability Computing Technology, Harbin \\ Engineering University, Harbin, Heilongjiang, 150001, P.R. China \\ ${ }^{2}$ National Laboratory for Information Science and Technology, Tsinghua \\ University, Beijing 100084, China \\ \{menchaoguang, luyongqian\} @hrbeu.edu.cn .
}

\begin{abstract}
In wireless sensor networks, an efficient Medium Access Control (MAC) protocol is critical, especially in terms of energy consumption and message latency. This paper introduces a novel protocol that nodes periodically sleep to conserve energy. When the source node has data packets to send, it uses a wakeup message to awake the sleeping nodes. The protocol needn't take the synchronization which must be achieved in S-MAC. It especially suits to the wireless sensor networks with narrow bandwidth.
\end{abstract}

Keywords: wireless sensor networks, media access control (MAC).

\section{Introduction}

Wireless sensor networks have emerged as one of the dominant technology trends of this decade (2000-2010) [1]. A wireless sensor network is comprised of a large number of tiny wireless senor nodes that are capable of sensing the environment and communicating in an ad-hoc fashion to deliver relevant information to the user. The small form factor of these nodes limits the battery life available for their operation. Furthermore, a critical event detected by the sensor network should be delivered to the user as soon as possible. Thus, for sensor networks, energy efficiency and latency both are the important parameters. In this paper, we present a novel medium access control protocol. It not only conserves energy through making the sensor nodes periodically sleep, but also has a low latency.

The rest of this paper is organized as follows: Section 2 analysis the defects of other MAC protocol in sensor networks, and describes our proposed protocol, An Adaptive Energy-efficient and Low-latency MAC Protocol (EL-MAC). Section 3 compares the performance of EL-MAC with existing mechanisms via simulations. Section 4 concludes the paper and presents our future work.

\section{An Adaptive Energy-Efficient and Low-Latency MAC Protocol}

In S-MAC [2], the periodic sleep may result in high latency, especially for multihop routing algorithms, because all the intermediate nodes have their own sleep schedules. 
The disadvantage of LAS-MAC [3] is that the nodes having data to forward must wait for next duty circle when they overhear interference, but the channel may not always be busy in this duty circle. So the throughput is reduced. The disadvantage of TWMAC [4] is that a busy tone must be transmitted before every data packet, this leads to energy waste and message latency. As mentioned, previous protocols have these defects. So a novel protocol with better performance is proposed in this paper. In our protocol, nodes periodically sleep to conserve energy, and the control messages are reduced on the premise that the reliable transmission is ensured. All of these cause energy conservation, latency decrease and throughput increase.

For brevity, we supposed that the carrier sensing range (radius) is one time larger than the transmission range[5-6]. Radios typically have four power levels corresponding to the following states: transmitting, receiving, listening, and sleeping. Typically, the power required to listen is about the same as the power to transmit and receive. The sleep power is usually one or four orders of magnitude less. Thus, a sensor should sleep as much as possible when it is not engaged in communication. EL-MAC make sensor nodes periodically sleep to conserve energy. Each node can be awaked by the wakeup signal at listen period.

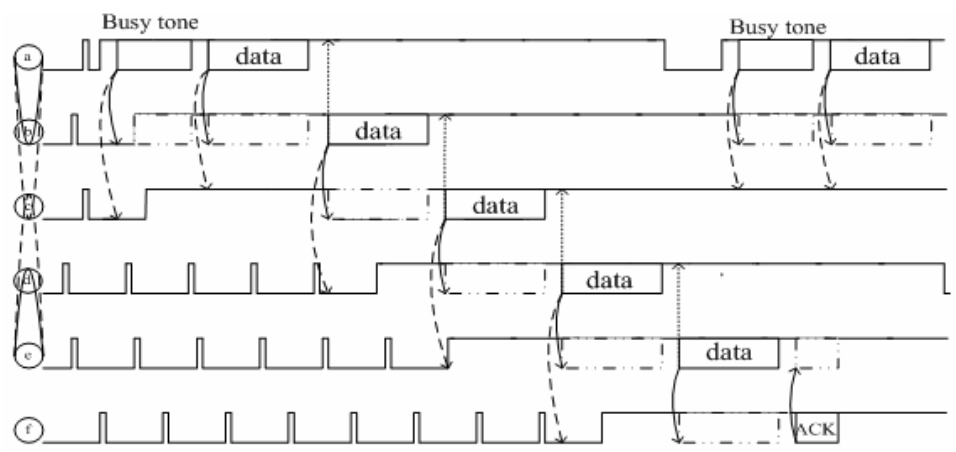

Fig. 1. Overview of EL-MAC

As shown in Fig.1, When a source node (node-a) has data to be sent to the sink node, It first sends a busy tone to wakeup its receiver (node-b). Nodes are not synchronized and, therefore, do not know the phase of each other's listen-sleep cycles in the listen mode. To avoid missing the short time that node-b has its radio on, the busy tone must be long enough to guarantee that all neighbors can sense the wakeup signal. When node-a sends the busy tone, its second hop neighbor (node-c) which is at the carrier sensing zone of node-a also can sense the wakeup signal. So node-b and nodec change their states to listening together. After the busy tone transmission, the source node listens for a short time. If the channel is idle, the data packet will be transmitted in succession. The data packet must be larger than the busy tone. If receiver, node-b, received the data packet successfully and it is not the sink node, it will forward the data packet to the next hop neighbor (node-c). Here, node-c has been waked up, so it can correctly receive that packet. Node-a will consider the data packet sent by node-b as the virtual ACK which denotes the data packet sent by node-a was successfully 
received by node- $b$, if not it repeats to transmit the packet. Then, if the packet needs to be forwarded further, the process is repeated until the sink node receives the data packet successfully and returns an ACK packet.

\section{Simulations and Performance Evaluation}

We run simulations using opnet. 100 nodes are deployed randomly in an area of 300 $\star 300 \mathrm{~m}^{2}$. We choose three source nodes and one sink node from the different corner.The radio range (radius) is $30 \mathrm{~m}$. We set the bandwidth to $20 \mathrm{kbps}$. Each data packet size is 250 bytes, and control packet size is 10 bytes. The busy tone packet size should be 50 bytes. We use the same energy consumption model as in [7] for the radio hardware. The simulation time is one hour.

We compare EL-MAC against S-MAC without the synchronization function. The energy consumption versus duty cycle is plotted in Fig.2. This result exactly demonstrates that EL-MAC can implement energy conservation task successfully.

Fig.3 shows the latency that data packets pass from a source node to a sink node. It shows that EL-MAC performs as better as 802.11 MAC on data packets passing latency. The reason is that the data packet passing of EL-MAC nearly doesn't have the sleep latency expounded in [2].

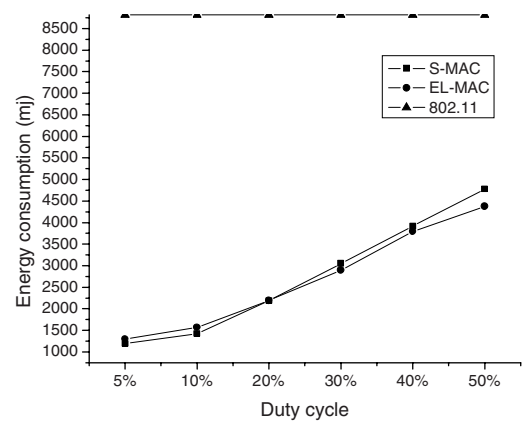

Fig. 2. Energy consumption analysis

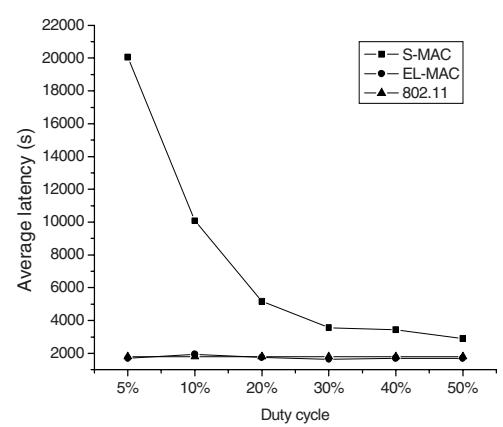

Fig. 3. Data latency analysis

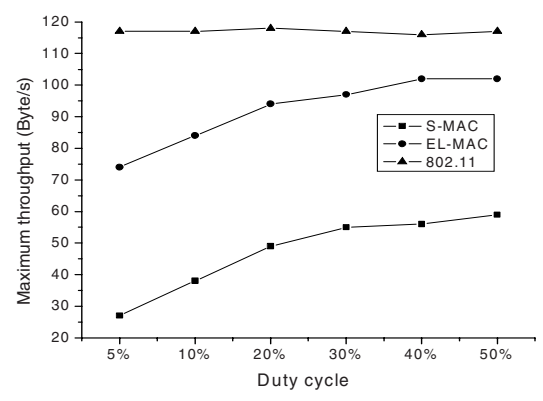

Fig. 4. Throughput analysis 
Fig. 4 shows the measured throughput for different duty cycle on the sink node. The busy tone becomes small when the duty cycle increases, this diminishes the cost of retransmission, in turn, diminishes the useless load of network. So EL-MAC throughput increases along with the duty cycle. As is shown, the EL-MAC have a much higher throughput than S-MAC.

\section{Conclusions and Future Work}

This paper presents a novel MAC protocol for wireless sensor networks. Compared to S-MAC, it makes following improvements: minimizing the latency of data passing, increasing the potential space of energy conserving.

Future work includes system scaling studies, parameter analysis and listen time decrease. More simulations will be done for the parameter analysis. The physical layer capability should be improved, so as to decrease the listen time.

\section{References}

1. Munee. Ali, Umar Saif, Adam Dunkels: Medium Access Control Issues in Sensor Networks, In: ACM SIGCOMM Computer Communication Review, (2006), 36(2):33-36.

2. Wei Ye, John Heidemann, Deborah Estrin: Medium Access Control with Coordinated Adaptive Sleeping for Wireless Sensor Networks, IEEE/ACM TRANSACTIONS ON NETWORKING, (2004), 12(3):493-506.

3. Jaesub Kim, Keuntae Park, Jeong-Hun Shin, Daeyeon Park: Look-Ahead Scheduling For Energy-Efficiency and Low-Latency in Wireless Sensor Networks, PE-WASUN'06, Spain, (2006), 10:141-144.

4. Matthew J. Miller, Nitin H. Vaidya: A MAC Protocol to Reduce Sensor Network Energy Consumption Using a Wakeup Radio, IEEE TRANSACTION ON MOBILE COMPUTING, (2005), 4(3):228-241.

5. Eun-Sun Jung, Nitin H. Vaidya: A Power Control MAC Protocol for Ad Hoc Networks, In: Proceedings of the IEEE/ACM MobiCom Conference, (2002), 9:36-47.

6. Kamerman A, Monteban L: WaveLAN-II: A High-Performance Wireless LAN for The Unlicensed Band, Bell Labs Technical Journal, (1997), 2(3):118-133.

7. Peng lin, Chunming Qiao, Xin Wang: Medium Access Control With a Dynamic Duty Cycle for Sensor Networks, in WCNC 2004/IEEE Wireless Communications and Networking Conference, (2004), 5(1):1522-1527. 